\title{
Application of next-generation sequencing to characterize novel mutations in clarithromycin-susceptible Helicobacter pylori strains with A2143G of $23 \mathrm{~S}$ rRNA gene
}

\author{
Jiaoe Chen ${ }^{1}$, Liping $\mathrm{Ye}^{2}$, Liangmin $\mathrm{Jin}^{1}$, Xuehua $\mathrm{Xu}^{1}$, Peisong $\mathrm{Xu}^{3}$, Xianjun Wang ${ }^{4}$ and Hongzhang $\mathrm{Li}^{\mathrm{i}^{*}}$
}

\begin{abstract}
Background: Clarithromycin (CLR) resistance has become a predominant factor for treatment failure of Helicobacter pylori eradication. Although the molecular mechanism of CLR resistance has been clearly understood in $\mathrm{H}$. pylori, it is lack of evidence of other genes involved in drug resistance. Furthermore, the molecular mechanism of phenotype susceptible to CLR while genotype of 23S rRNA is mutant with A2143G is unclear. Here, we characterized the mutations of CLR-resistant and -susceptible H. pylori strains to explore bacterial resistance.
\end{abstract}

Methods: In the present study, the whole genomes of twelve clinical isolated H. pylori strains were sequenced, including two CLR-susceptible strains with mutation of A2143G. Single nucleotide variants (SNVs) were extracted and analyzed from multidrug efflux transporter genes.

Results: We did not find mutations associated with known CLR-resistant sites except for controversial T2182C outside of A2143G in the 23S rRNA gene. Although total SNVs of multidrug efflux transporter gene and the SNVs of HP0605 were significant differences $(P \leq 0.05)$ between phenotype resistant and susceptible strains. There is no significant difference in SNVs of RND or MFS (HP1181) family. However, the number of mutations in the RND family was significantly higher in the mutant strain (A2143G) than in the wild type. In addition, three special variations from two membrane proteins of mtrC and hefD were identified in both CLR-susceptible strains with A2143G.

Conclusions: Next-generation sequencing is a practical strategy for analyzing genomic variation associated with antibiotic resistance in $H$. pylori. The variations of membrane proteins of the RND family may be able to participate in the regulation of clinical isolated $H$. pylori susceptibility profiles.

Keywords: Helicobacter pylori, Clarithromycin, Susceptible strains, 23S rRNA, SNVs, RND family

\section{Background}

Helicobacter pylori (H. pylori), a Gram-negative and microaerophilic bacterium, has been recognized an important human pathogen that infects approximately $50 \%$ of world's population, and is responsible for the development of upper gastrointestinal disorders, including chronic gastritis, peptic ulcer disease, gastric cancer

\footnotetext{
*Correspondence: smyylhz@163.com

1 Department of Gastroenterology, Sanmen People's Hospital, No. 117, Renmin Road, Sanmen 317100, Zhejiang, People's Republic of China

Full list of author information is available at the end of the article
}

and mucosa-associated lymphoid tissue (MALT) lymphoma [1-3]. In the past few decades, triple therapy regimen consist of a proton pump inhibitor in combination with two antibiotics, such as clarithromycin (CLR) and amoxicillin (AMX) or metronidazole (MTZ), which has been recommended as first-line treatment regimen for $H$. pylori infection [4]. However, with the increase of $H$. pylori CLR-resistant strains, this traditional treatment regimen is being replaced by quadruple therapy or precise medical, especially in the area of CLR resistance is higher than 15\% [5-7]. Many reports have indicated that 
CLR resistance has become a predominant factor for treatment failure in which containing CLR $[5,8]$.

The majority of $H$. pylori CLR-resistant strains present three point mutations in the region of domain $\mathrm{V}$ of $23 \mathrm{~S}$ ribosomal RNA (rRNA): A2142G, A2142C and A2143G. Simultaneously, the studies also suggest that some other point mutations may be involved in CLR resistance at position 2115G, G2141A, T2117C, T2182C, T2717C [9-11]. Another mechanism of resistance to CLR has been reported that five conserved families of multidrug efflux pump transporter contribute to bacterial antibiotic resistance. One of these, the resistance-nodulation-cell division (RND) family, was consisted of an inner membrane efflux protein, a membrane fusion protein and an outer membrane protein. Currently, four gene clusters (HP0605-HP0607, HP0969-HP0971, HP1327-HP1329, HP1487-HP1489) [12-15] have been established as RND family candidates in $H$. pylori. Hirata et al. reported that the MIC of CLR-resistant strains was decreased by using efflux pump inhibitor (EPI), indicating that in addition to the point mutation of $23 \mathrm{~S}$ rRNA gene, the efflux pump cluster is also involved in the development of resistance to CLR [16].

Although the molecular mechanism of CLR resistance has been relatively clearly understood in $H$. pylori, it is unclear whether other gene mutations associated with CLR resistance outside 23S rRNA and RND family. With increase of the CLR-resistant strains, several studies have suggested that other genetic factors could be involved in the increased antibiotic resistance. Recently, Binh et al. [17] revealed that mutations of insertion or deletion in rpl22 and guanine to adenine point mutation at position 60 in $\operatorname{infB}$ gene could be related to CLR resistance using whole-genome sequencing of induced CLR-resistant strains in vitro.

However, almost all of the researches were focus on exploring potential antibiotic resistance genes. Until now, to the best of our knowledge, there is very little research on antibiotic susceptibility gene. In our previous research, we isolated two strains that were identified adenine to guanine mutations at position 2143 in $23 \mathrm{~S}$ rRNA, while the outcome of antibiotic susceptibility testing were susceptibility to CLR or lower CLR resistance, suggesting that there were some other genes participated in the regulation of antibiotic resistance or susceptibility to CLR.

Compared with traditional DNA sequencing, nextgeneration sequencing (NGS) is a revolution of sequencing technology that can be sequencing millions of DNA molecules massively parallel in less time and at lower cost $[18,19]$. To date, more than three thousand microbial genome sequences have been completed and published by using NGS according to the report [20,21]. Now, NGS has also been used to identify bacterial single nucleotide polymorphisms (SNP) or mutation associated with antibiotic resistance [22-24]. Although we didn't identify all of sequencing information that is like a needle in a haystack because a large number of redundant data is generated by the NGS, with the development of sequencing and biological information, the genetic code will be eventually cracked one by one.

In this study, to characterize the multidrug efflux transporter gene variants in the CLR genotype-resistant while phenotype-susceptible strains, we applied Sanger sequencing to detect the genotype of $23 \mathrm{~S}$ rRNA and NGS to analysis of genomic variation in clinical isolated $H$. pylori strains. CLR susceptibility testing was performed by E-test and agar dilution. Ten HP strains meeting our requirements were applied to whole-genome sequencing, including four CLR-resistant and six CLR-susceptible strains.

\section{Methods}

\section{Isolation and culture of $H$. pylori}

Gastric mucosa tissue samples were collected from patients with upper gastrointestinal disease during endoscopy at Sanmen People's Hospital and Zhejiang Taizhou Hospital. Isolation and culture of $H$. pylori were performed at the laboratory of Hangzhou Zhiyuan Medical Inspection Institute. Patients were investigated to have not taken any antibiotics for at least 4 weeks before examination. This study had received a strict medical ethics review, and written informed consent was obtained from every patient.

The isolation and identification of $H$. pylori were performed as described in previous studies [25, 26]. Gastric mucosa homogenate tissue was transferred onto a Columbia agar plates containing $5 \%$ fresh defibrinated sheep blood and cultured under microaerophilic conditions $\left(5 \% \mathrm{O}_{2}, 10 \% \mathrm{CO}_{2}\right.$ and $\left.85 \% \mathrm{~N}_{2}\right)$ at $37^{\circ} \mathrm{C}$ for $3-7$ days. Suspicious colonies were confirmed by Gram stain, urease, oxidase, and catalase activity testing.

\section{Antibiotic susceptibility testing}

The antibiotic resistance of $H$. pylori to CLR was performed by E-test and agar dilution methods according to the protocols of the Clinical and Laboratory Standards Institute (Wayne, PA, USA) [27]. Briefly, the concentration of $H$. pylori was regulated with saline to a 2.0 McFarland standard, and the suspensions were inoculated onto Mueller-Hinton agar plate supplemented with 5\% sheep blood. The CLR E test strip was attached on the plate and incubated at $37{ }^{\circ} \mathrm{C}$ for $3-5$ days under microaerophilic conditions. Agar dilution was performed by serial twofold dilutions of CLR. The breakpoint of CLR resistance was $\geq 1 \mathrm{mg} / \mathrm{l}$. ATCC43504 (NCTC11637) was used as the 
control strain and all tests were performed by Hangzhou Zhiyuan Medical Inspection Institute.

\section{Direct sequencing characterized the mutations of $23 \mathrm{~S}$ rRNA}

According to the reference sequence of HP U27270, HP-23S forward primer (5'-ATGAATGGCGTAACG AGATG-3') and HP-23S reverse primer (5'-ACACTC AACTTGCGATTTCC-3') were employed to detect $23 \mathrm{~S}$ rRNA gene mutations at positions of 2142 and 2143. The PCR reaction was performed in $25-\mu \mathrm{l}$ reactions containing $2.5 \mu \mathrm{l}$ of $10 \times \mathrm{LA}$ Taq Buffer, $4 \mu \mathrm{l}$ of dNTP mixture (2.5 mM each), $0.5 \mu \mathrm{l}$ each $10 \mu \mathrm{M}$ primer, $2 \mu \mathrm{l}$ template DNA and $0.25 \mu \mathrm{l}$ of TaKaRa LA Taq ${ }^{\mathrm{TM}}$ ( 5 units/ $\left.\mu \mathrm{l}\right)$. The parameters of PCR were carried out at $94{ }^{\circ} \mathrm{C}$ for $5 \mathrm{~min}$, followed by 25 cycles of denaturing at $94{ }^{\circ} \mathrm{C}$ for $30 \mathrm{~s}$, annealing at $58{ }^{\circ} \mathrm{C}$ for $30 \mathrm{~s}$, and extending at $72{ }^{\circ} \mathrm{C}$ for $3 \mathrm{~min}$, with a final extension for $10 \mathrm{~min}$ at $72{ }^{\circ} \mathrm{C} .1 .2 \%$ agarose gel electrophoresis was utilized for verifying the PCR products size at $360 \mathrm{bp}$. To validate the mutations of $23 \mathrm{~S}$ rRNA, Sanger sequencing was performed with an ABI 3730XL DNA Analyzer (Applied Biosystems, Foster City, CA, USA) using BigDye ${ }^{\circledR}$ Terminator V3.1 according to the manufacturer's instructions.

\section{DNA extraction and whole-genome sequencing}

The total genomic DNAs of $H$. pylori were extracted by using Invitrogen Purelink Genomic DNA Mini Kit (Life Technologies, Carlsbad, CA, USA) according to the manufacturer's instructions. The concentration of each genomic DNA sample was quantified with Qubit dsDNA HS assay kit (Life Technologies). For each sample, $1 \mu \mathrm{g}$ genomic DNA was randomly uniformly fragmented to $<500$ bp by sonication (Diagenode Bioruptor UCD-200) and the library was prepared by using NEB Next $^{\circledR}$ Ultra $^{\mathrm{TM}}$ DNA Library Prep Kit for Illumina ${ }^{\circledR}$ following the manufacturer's protocol. Quality control of the library was identified by Agilent 2100 Bioanalyzer (Agilent Technologies, Palo Alto, CA, USA) with DNA 1000 chip according to the manufacturer's instructions. Whole-genome sequencing was performed with the Illumina HiSeq (Illumina, San Diego, CA, USA) platform to generate $2 \times 150$-bp paired-end reads. Image analysis and base calling were conducted by the HiSeq Control Software (HCS) and GAPipeline-1.6 (Illumina) on the HiSeq instrument.

\section{Genome analysis and single nucleotide variations (SNVs) calling}

To reduce the false discovery rate of SNVs, the lowquality bases (the quality of both ends bases $<$ Q20) and raw reads ( $>10 \mathrm{~N}$ bases and reads lengths $<75 \mathrm{bp}$ ) were trimmed by Trimmomatic (version 0.30 ). The remaining clean reads were mapped against the reference genome with GenBank accession NC_000915 and CP003904 using BWA [28] (version 0.7.12). SNVs and InDel were called using SAMTOOLS's [29] (version 1.1) Mpileup module and Bcftools with default parameters, and finally a series of mutations were generation.

\section{Variations of multidrug resistance genes}

In order to validate the role of multidrug efflux transporter gene in CLR-resistant and CLR-susceptible $H$. pylori, the variations of RND family, major facilitator superfamily (MFS) and adenosine triphosphate (ATP)binding cassette $(\mathrm{ABC})$ superfamilies had been analyzed, including HP0605-HP0607, HP0969-HP0971, HP1327HP1329, HP1487-HP1489, HP1181, HP1184, HP0600, HP0613, HP0759, HP1082, HP1206, HP1220, HP1321 and HP1486.

\section{Statistical analysis}

All statistical analyses were conducted with SPSS statistical software package version 19.0 (SPSS Inc., Chicago, IL, USA). The relationships between variations of multidrug resistance genes and CLR resistance/susceptibility were investigated by Student's t-test. A P value $\leq 0.05$ was considered statistically significant.

\section{Results}

Genotype of $23 \mathrm{~S}$ rRNA and phenotype of $H$. pylori resistant to CLR

For twelve clinical isolated strains, CLR susceptibility testing indicated that the six of strains were susceptible to CLR and six of strains were resistant to CLR. The genotype of $23 \mathrm{~S}$ rRNA from $H$. pylori was verified by Sanger sequencing (Fig. 1). Eight of them were mutant with A2143G and four strains were wild type (Table 1). All of phenotype-resistant strains presented mutation A to $G$ at position 2143 of the $23 \mathrm{~S}$ rRNA. However, two mutanttype (S2, S3) of 23S rRNA gene at position 2143 (A>G) were also detected in six phenotype-susceptible strains.

\section{Overview of whole-genome sequencing of clinical isolated H. pylori}

In the present study, twelve strains of $H$. pylori genome were successfully figured out by Hiseq sequencer. After trimming the low-quality reads, clean reads ranged from 6.6 to 12.2 million (Table 1 ). Clean reads were directly mapped to the reference genome. Coverage and average depth ranged from 90.98 to $93.16 \%$ and from 378 to 888 , respectively. Therefore, the efficient reads were sufficient for subsequent analysis of SNVs. The number of SNVs and InDels ranged from 67,219 to 70,933 and from 67 to 177 , respectively. There were no significant differences between CLR-resistant and CLR-susceptible strains 
a

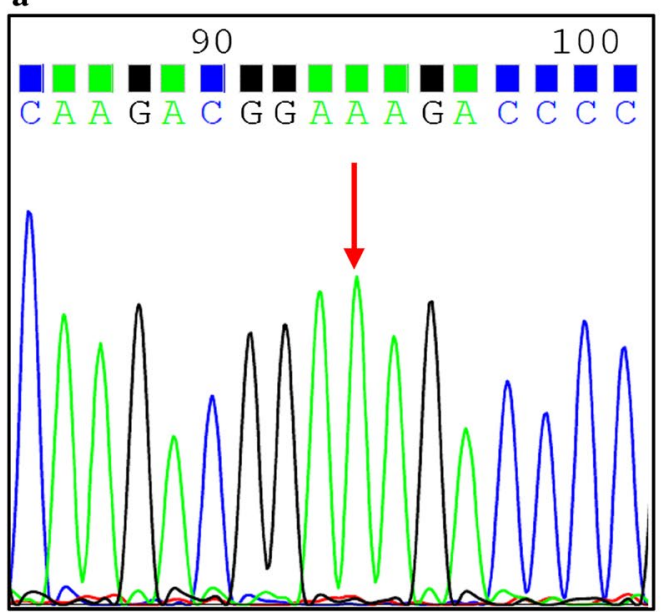

b

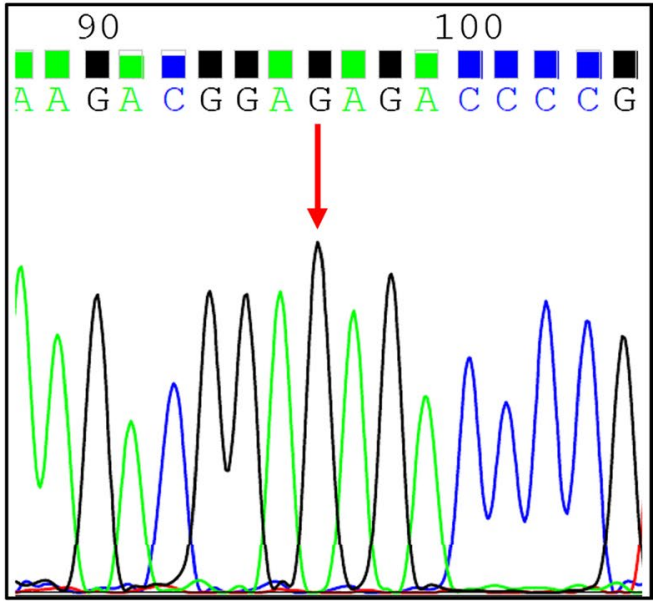

Fig. 1 The results of Sanger sequencing for genotype of $23 \mathrm{~S}$ rRNA in H. pylori. a The wild type without mutation at position 2143. b The mutant strain with mutation of $A>G$ at position 2143

Table 1 The results of whole-genome sequencing for each sample

\begin{tabular}{|c|c|c|c|c|c|c|c|c|}
\hline Strain ID & $\begin{array}{l}\text { CLR susceptibil- } \\
\text { ity testing }\end{array}$ & $\begin{array}{l}\text { Total reads } \\
\text { (clean reads) }\end{array}$ & $\begin{array}{l}\text { Mapping } \\
\text { to genome } \\
\text { reads }\end{array}$ & Covered length & Coverage (\%) & Average depth & Total SNVs & Total InDels \\
\hline S1 & $\mathrm{R}^{\mathrm{a}}$ & $9,815,508$ & $8,780,946$ & $1,517,430$ & 90.98 & 545.66 & 67,219 & 106 \\
\hline S2 & $S^{b}$ & $10,573,646$ & $9,813,458$ & $1,534,369$ & 91.99 & 601.38 & 68,671 & 95 \\
\hline S3 & $S$ & $10,269,810$ & $9,529,590$ & $1,527,013$ & 91.55 & 565.38 & 70,135 & 101 \\
\hline S4 & $\mathrm{R}$ & $9,931,112$ & $8,921,164$ & $1,553,847$ & 93.16 & 522.64 & 67,929 & 132 \\
\hline S5 & $\mathrm{R}$ & $9,965,800$ & $8,012,304$ & $1,522,585$ & 91.29 & 459.11 & 67,580 & 134 \\
\hline S6 & S & $8,326,490$ & $7,203,062$ & $1,537,013$ & 92.15 & 528.19 & 68,032 & 99 \\
\hline S7 & S & $8,023,030$ & $7,541,380$ & $1,536,514$ & 92.12 & 549.71 & 68,496 & 112 \\
\hline S8 & $\mathrm{R}$ & $7,687,812$ & $7,171,310$ & $1,522,399$ & 91.28 & 535.58 & 69,891 & 104 \\
\hline S9 & $\mathrm{S}$ & $11,190,968$ & 9805694 & $1,522,469$ & 91.28 & 731.93 & 68,658 & 67 \\
\hline$S 10$ & S & $12,167,520$ & $11,643,382$ & $1,539,435$ & 92.3 & 888.02 & 70,933 & 94 \\
\hline S11 & $\mathrm{R}$ & $9,756,502$ & $8,630,028$ & $1,517,874$ & 91.01 & 532.33 & 67,285 & 112 \\
\hline $\mathrm{S} 12$ & $\mathrm{R}$ & $6,575,708$ & $6,090,808$ & $1,521,421$ & 91.22 & 378.89 & 67,231 & 177 \\
\hline
\end{tabular}

S2 and S3 (italic values) indicates the phenotype-susceptible strains with mutant in A2143G of 23S rRNA gene

${ }^{a} \mathrm{R}$ indicated the strain was resistant to clarithromycin

b $S$ indicated the strain was susceptible to clarithromycin

in SNVs despite more prevalent in CLR-susceptible strains. However, the number of InDels was significantly decreased in CLR-susceptible strains.

\section{Identification of 23S rRNA gene mutations}

To analyze the associations between phenotypic resistance and genotypic resistance, the mutations of the 23S rRNA gene were investigated. Consistent with Sanger sequencing, whole-genome sequencing indicated that six phenotype-resistant strains and two phenotypesusceptible strains had mutation $A>G$ at position 2143. Simultaneously, the mutations outside of 2143 had been extracted (Table 2). Totally, we found fourteen mutations in addition to 2143. In this study, we did not find any mutations associated with known CLR-resistant sites except for T2182C and there is no significant difference between resistant strain and susceptible strain regardless of whether there was mutant with A2143G.

\section{Identification of multidrug efflux transporter gene mutations}

To characterize the mutations of multidrug efflux transporter genes, we focused on the study of the RND family, MFS and ABC superfamilies in H. pylori. Prior to 
Table 2 SNVs of the 23S rRNA outside of 2143

\begin{tabular}{|c|c|c|c|c|c|c|c|c|c|c|c|c|c|c|}
\hline Nucleotide position & Ref & Mutation & S1 & S2 & S3 & S4 & S5 & S6 & S7 & S8 & S9 & S10 & S11 & $\mathrm{S} 12$ \\
\hline 973 & G & T & + & - & - & + & - & - & - & - & - & + & - & - \\
\hline 973 & G & A & - & - & - & - & + & - & - & - & + & - & - & - \\
\hline 973 & G & C & - & + & + & - & - & + & - & + & - & - & + & - \\
\hline 1023 & G & $A$ & + & - & + & - & - & - & - & - & + & - & - & - \\
\hline 1279 & A & T & - & - & - & - & - & - & + & - & - & - & - & - \\
\hline 1280 & A & G & - & - & - & - & - & - & + & - & - & - & - & - \\
\hline 1314 & G & A & + & - & - & - & + & - & - & + & - & - & - & - \\
\hline 1513 & G & A & + & + & - & - & - & - & + & - & + & - & - & - \\
\hline 2173 & $C$ & $\mathrm{~T}$ & + & - & - & - & + & - & - & + & - & - & - & - \\
\hline 2182 & $\mathrm{~T}$ & C & + & + & + & + & + & + & - & + & + & + & + & + \\
\hline 2302 & A & G & + & - & - & - & - & - & - & - & - & - & + & - \\
\hline 2485 & $\mathrm{~T}$ & $C$ & - & - & - & - & + & - & - & - & - & - & - & + \\
\hline 2143 & $A$ & G & + & + & + & + & + & - & - & + & - & - & + & + \\
\hline
\end{tabular}

S2 and S3 (italic values) indicates the phenotype-susceptible strains with mutant in A2143G of 23S rRNA gene

+ Represented that the mutation had been detected and - represented the mutation had not been detected

identification of gene mutation in multidrug efflux genes, we removed the synonymous and InDels mutations in the CDS region. All mutations of these genes were presented in Table 3. Regardless of whether the strain is resistant to CLR, gene mutations were detected in all $H$. pylori of multidrug efflux transporter genes. The gene mutations of membrane fusion proteins (HP0606, HP0970, HP1328, HP1488) were significantly less than

Table 3 SNVs of multidrug efflux pump transporter genes

\begin{tabular}{|c|c|c|c|c|c|c|c|c|c|c|c|c|}
\hline \multirow[t]{2}{*}{ Gene } & \multicolumn{12}{|c|}{ Strain } \\
\hline & S1 & $S 2$ & S3 & S4 & S5 & S6 & S7 & S8 & S9 & S10 & S11 & $\mathrm{S} 12$ \\
\hline HP0605 & 21 & 18 & 23 & 24 & 23 & 16 & 20 & 27 & 15 & 17 & 29 & 27 \\
\hline HP0606 & 5 & 5 & 6 & 1 & 4 & 7 & 5 & 6 & 8 & 5 & 2 & 8 \\
\hline HP0607 & 38 & 34 & 32 & 32 & 33 & 30 & 22 & 31 & 32 & 32 & 27 & 36 \\
\hline HP0969 & 26 & 23 & 25 & 25 & 24 & 25 & 26 & 23 & 26 & 26 & 24 & 23 \\
\hline HP0970 & 7 & 9 & 11 & 7 & 7 & 9 & 8 & 8 & 8 & 9 & 9 & 7 \\
\hline HP0971 & 11 & 15 & 12 & 11 & 13 & 12 & 14 & 11 & 8 & 9 & 10 & 11 \\
\hline HP1327 & 22 & 22 & 21 & 24 & 22 & 22 & 21 & 22 & 23 & 25 & 23 & 22 \\
\hline HP1328 & 15 & 12 & 15 & 14 & 12 & 14 & 13 & 14 & 15 & 14 & 12 & 14 \\
\hline HP1329 & 28 & 29 & 36 & 30 & 28 & 25 & 33 & 30 & 26 & 24 & 32 & 30 \\
\hline HP1487 & 22 & 21 & 15 & 20 & 21 & 18 & 16 & 18 & 18 & 19 & 17 & 16 \\
\hline HP1488 & 7 & 4 & 3 & 6 & 4 & 4 & 5 & 6 & 5 & 4 & 4 & 5 \\
\hline HP1489 & 25 & 28 & 23 & 22 & 23 & 25 & 19 & 20 & 24 & 29 & 25 & 19 \\
\hline HP0600 & 50 & 3 & 1 & 52 & 48 & 23 & 20 & 46 & 5 & 31 & 47 & 44 \\
\hline HP0613 & 9 & 7 & 6 & 9 & 7 & 15 & 3 & 9 & 10 & 15 & 8 & 5 \\
\hline HP0759 & 10 & 11 & 10 & 10 & 11 & 11 & 8 & 10 & 11 & 9 & 8 & 11 \\
\hline HP1082 & 10 & 13 & 12 & 10 & 11 & 12 & 17 & 11 & 15 & 11 & 12 & 16 \\
\hline HP1181 & 10 & 9 & 11 & 9 & 10 & 7 & 11 & 12 & 12 & 9 & 9 & 12 \\
\hline HP1184 & 14 & 19 & 14 & 22 & 15 & 14 & 12 & 18 & 13 & 14 & 17 & 15 \\
\hline HP1206 & 13 & 16 & 14 & 13 & 14 & 13 & 27 & 16 & 16 & 18 & 18 & 12 \\
\hline HP1220 & 3 & 3 & 4 & 3 & 2 & 1 & 4 & 3 & 1 & 1 & 2 & 1 \\
\hline HP1321 & 26 & 30 & 29 & 22 & 24 & 29 & 32 & 30 & 24 & 24 & 30 & 28 \\
\hline HP1486 & 21 & 18 & 18 & 18 & 18 & 17 & 16 & 17 & 18 & 15 & 17 & 12 \\
\hline Total & 393 & 349 & 341 & 384 & 374 & 349 & 352 & 388 & 333 & 360 & 382 & 374 \\
\hline
\end{tabular}

S2 and S3 (italic values) indicates the phenotype-susceptible strains with mutant in A2143G of 23S rRNA gene 
inner membrane proteins or outer membrane proteins in RND family.

We didn't find significant differences in gene mutations of RND or MFS (HP1181) families between CLR phenotype resistant strains and CLR phenotype susceptible strains. However, the total SNVs of multidrug efflux genes were significant differences between them. Unexpectedly, when we were grouped according to the 23S rRNA genotype, the number of mutations in the RND family was significantly higher in the mutant strain (A2143G) than in the wild type, and the difference was statistically significant. These results suggested the mutations from multidrug efflux genes may play an important role in CLR-resistance, and the mutations of the RND family may change the resistance of $H$. pylori to CLR with A2143G.

Special variations in CLR-susceptible strains with A2143G In the present study, we found two $H$. pylori strains were susceptible to CLR, while the genotypes of $23 \mathrm{~S}$ rRNA were mutant with A2143G. To understand the cause of this discrepancy, analysis of the special variations in both strains was performed. The special variations were extracted by removing the mutations present in other CLR resistant strains with mutation of A2143G. Totally, 320 non-synonymous variations were obtained, and we selected 16 variations in 14 genes as putative susceptible gene (Table 4).

Variations were mainly concentrated in membrane proteins and $\mathrm{ABC}$ transport ATP-binding proteins. Membrane proteins of the RND family, including $m t r C$ and hefD, were identified variations in $H$. pylori CLRsusceptible strains with mutation of A2143G. The variations of major facilitator superfamily of HP1181 and ABC superfamilies were found in both strains. Simultaneously, two terminate mutations were observed in Hydrogenase maturation factor (hypD) and molybdenum cofactor biosynthesis protein A (moaA).

\section{Discussion}

CLR has been widely used as a first-line drug for $H$. pylori eradication therapy, and has achieved remarkable achievements for the past few decades [4, 30]. Although the mechanism of CLR resistance has been well illustrated, it is difficult to explain the bacterial antibiotic resistance of some strains with different genotype and phenotype. However, researchers were mainly committed to exploring the genes associated with CLR resistance $[11,17]$. To our best knowledge, there is little attention on CLR susceptible gene. In this study, to characterize the variations of multidrug efflux transporter genes, we first complete the whole-genome sequencing of CLRsusceptible $H$. pylori with mutation of A2143G in the $23 \mathrm{~S}$ rRNA.

In the present study, we applied HiSeq 2500 platform to generate sufficient reads for analyzing the whole genome sequences of $H$. pylori. The clean short overlapping reads after quality control were directly mapped against reference genome without assembly [31]. Although the coverage was not very high, the multidrug efflux transporter genes and 23S rRNA gene were well covered with depth

Table 4 The special variations were identified in CLA-resistant strain with A2143G

\begin{tabular}{|c|c|c|c|c|c|c|}
\hline Gene & Position of mutation $^{a}$ & Ref & Mutation & Amino acid & Annotation & Mean depth \\
\hline HP0254 & 265 & G & A & G89S & Outer membrane protein HopG & 465.5 \\
\hline rpsA & 482 & G & A & G161D & 305 ribosomal protein $\$ 1$ & 543.5 \\
\hline mtrC & 542 & C & $\mathrm{T}$ & $\mathrm{T} 1811$ & Membrane fusion protein & 525.5 \\
\hline HP0498 & 744 & $\mathrm{~T}$ & G & F248L & Sodium $^{-}$and chloride ${ }^{-}$dependent transporter & 597.5 \\
\hline prowX & 1650 & A & C & R550S & Osmoprotectant ABC transporter permease & 642 \\
\hline HP0853 & 943 & C & A & R315S & ABC transporter ATP-binding protein & 441.5 \\
\hline hypD & 474 & G & A (stop) & W158X & Hydrogenase maturation factor & 512 \\
\hline hefD & 13 & G & $\mathrm{T}$ & G5C & Outer membrane protein & 583.5 \\
\hline hefD & 82 & A & G & $\mathrm{M} 28 \mathrm{~V}$ & Outer membrane protein & 346 \\
\hline tolB & 210 & $\mathrm{~T}$ & G & D70E & Translocation protein & 705.5 \\
\hline rpsP & 226 & G & $A$ & A76T & $30 S$ ribosomal protein S16 & 650 \\
\hline HP1181 & 826 & A & G & I276V & Multidrug transporter & 506 \\
\hline rplA & 464 & G & A & S155N & $50 S$ ribosomal protein L16 & 561.5 \\
\hline HP1220 & 661 & G & A & $\mathrm{A} 221 \mathrm{~T}$ & ABC transporter ATP-binding protein & 628 \\
\hline HP1220 & 662 & C & $\mathrm{T}$ & A221V & ABC transporter ATP-binding protein & 624.5 \\
\hline moaA & 76 & $C$ & $\mathrm{~T}$ (stop) & Q26X & Molybdenum cofactor biosynthesis protein A & 528.5 \\
\hline
\end{tabular}

${ }^{a}$ Nucleotide position was determined by the respective reference sequence from NC_000915 
of at least 300-fold. Because of genomic gaps, we didn't choice to analysis of insertions or deletions (InDels).

To elucidate the differences in genotype and phenotype, the analysis of $23 \mathrm{~S}$ rRNA gene mutation was carried out, and the point mutations at position 2143 were consistent with NGS. Consequently, NGS was a precise method to distinguish the point mutation in genome [22-24]. Overall, mutations of 23S rRNA were disorganized and unregulated. There were no significant differences between CLR phenotype-resistant strains and CLR phenotype-susceptible strains, and nor between CLR genotype-resistant strains and CLR genotype-susceptible strains. On the contrary, the mutation of T2182C was detected in all strains except for S7. Although gene mutation of T2182 was identified with low resistance level in previous study [32], this mutation was detected in most of the strains in China and the result of this discrepancy may be contribute to geographical and genetic factors [2].

In present study, to analyze the discrepancy between CLR-resistant strains and CLR-susceptible strains, the mutations of twenty-two multidrug efflux transporter genes were extracted. HP0605 knockout mutant presents more susceptibility to novobiocin and sodium deoxycholate [12]. Agreement with previous study, we found that the total SNVs of multidrug efflux transporter gene and the SNV of HP0605 were significant differences $(\mathrm{P} \leq 0.05)$ between phenotype resistant and susceptible strains, while not been found in other genes [33]. Thus, we speculate the SNVs of HP0605 probably have an effect on $H$. pylori resistance to CLR. Unexpectedly, the SNVs of the RND family were significantly higher in mutant strain (A2143G) than in wild type. The different results mainly contributed to the two strains with different genotype and phenotype. We speculated that the 23S rRNA mutant strains with susceptible to CLR were initially resistant to CLR. Nonetheless, with the living environment and genetic changes, the phenotype of CLR has transformed. This can illustrate that these two strains exhibit more tendency to other strains with consistent genotype and phenotype in genetics.

Efflux pump systems have been identified in bacteria to be associated with antibiotic resistance [14-16]. The RND family, commonly used as a Gram-negative bacteria antibiotic study, is also used for $\mathrm{H}$. pylori. Amsterdam et al. [12] revealed that more susceptible to metronidazole (MTZ) for HP0605 and HP0971 double-knockout mutant $H$. pylori strain. The expression of membrane proteins of the RND family has been a hot spot in the study of bacterial resistance. It has been proven that TolC and its homologues play an important role in molecules efflux, virulence and drug resistance [34]. hefD (HP0971) and $m \operatorname{trC}$ (HP0606) are an outer membrane protein (TolC) and a membrane fusion protein (AcrA) of the
RND family, respectively. The result of NGS has shown that there have three special mutations present in two CLR-susceptible strains with genotype of A2143G, and not in others. And also, this phenomenon has been found in outer membrane protein hopG (HP0254). Therefore, we attempt to speculate that the variations of outer membrane protein are associated with bacterial antibiotic resistance in $H$. pylori. Although the exact molecular mechanism of $H$. pylori with genotype of A2143G susceptible to CLR is unclear, our findings have indicated that the variations of membrane proteins possibly contribute to influence $H$. pylori resistance to CLR.

Of course, our research also has some limitations due to the problem of coverage and we did not analyze the impact of InDels on the experimental results. We empirically selected some genes that were associated with drug resistance for SNVs analysis. Indeed, we have characterized some special variations of HP0606 and HP0971 in CLR-susceptible strains with mutation of A2143G, and we believed that there have some other genes that regulate the susceptibility of bacteria to antibiotics. Undeniably, whole-genome sequencing of $H$. pylori provides a new way to solve the problem of bacteria antibiotic resistance.

\section{Conclusion}

In this study, we successfully isolated two CLR-susceptible $H$. pylori strains with mutation A2143G of 23S rRNA gene. Genome variations were analyzed by whole-genome sequencing between twelve $H$. pylori strains with different CLR resistances. The data of sequencing suggested that the next-generation sequencing of clinical isolated H. pylori is a useful method for identifying genome variations. Analysis of multidrug efflux transporter gene mutation results indicated that membrane proteins of RND family possibly play an indispensable role in resistance to CLR. Further studies of $H$. pylori genomic variation should be paid more attention to disentomb potential gene associated with antibiotic resistance or susceptibility.

\section{Abbreviations}

SNVs: single nucleotide variants; CLR: clarithromycin; RND: resistance-nodulation-cell division; NGS: next-generation sequencing; MFS: major facilitator superfamily; InDels: insertions or deletions.

\section{Authors' contributions}

$\mathrm{HL}$ conceived and designed the study. JC, LY, LJ, XX and XW contributed to collected samples. PX performed the laboratory tests. HL, JC, LY and PX participated in analyzing data and writing the manuscript. All authors read and approved the final manuscript.

\section{Author details}

${ }^{1}$ Department of Gastroenterology, Sanmen People's Hospital, No. 117, Renmin Road, Sanmen 317100, Zhejiang, People's Republic of China. ${ }^{2}$ Department of Gastroenterology, Zhejiang Taizhou Hospital, Taizhou 31700, People's Republic of China. ${ }^{3}$ Department of Research Service, Zhiyuan Inspection 
Medical Institute, Hangzhou, Zhejiang 310006, People's Republic of China. ${ }^{4}$ Clinical Laboratory, Hangzhou First People's Hospital, Hangzhou 310006, Zhejiang, People's Republic of China.

\section{Acknowledgements}

We thank Fei Meng for excellent technical assistance and much appreciate Yunhui Liu for proofreading of manuscript.

\section{Competing interests}

The authors declare that they have no competing interests.

\section{Availability of data and materials}

The strains and sequencing data are available from the corresponding author on reasonable request.

\section{Consent for publication}

Not applicable.

\section{Ethics approval and consent to participate}

This study had received a strict medical ethics review. Written informed consent was obtained from the patients.

\section{Funding}

This study was financially supported by Medical and health plan of Zhejiang Province (2015DTA020), Public Technology Application Research of Zhejiang Province Science and Technology Hall (2017C33192 and 2016C33232) and Natural Science Foundation of Zhejiang Province (LY13H190003).

\section{Publisher's Note}

Springer Nature remains neutral with regard to jurisdictional claims in published maps and institutional affiliations.

Received: 16 March 2017 Accepted: 8 March 2018

Published online: 22 March 2018

\section{References}

1. McColl KE. Clinical practice. Helicobacter pylori infection. N Engl J Med. 2010;362:1597-604.

2. Fock KM, Ang TL. Epidemiology of Helicobacter pylori infection and gastric cancer in Asia. J Gastroenterol Hepatol. 2010;25:479-86.

3. Park JB, Koo JS. Helicobacter pylori infection in gastric mucosa-associated lymphoid tissue lymphoma. World J Gastroenterol. 2014;20:2751-9.

4. Malfertheiner P, Megraud F, O'Morain C, Bazzoli F, El-Omar E, Graham D, Hunt R, Rokkas T, Vakil N, Kuipers EJ. Current concepts in the management of Helicobacter pylori infection: the Maastricht III Consensus Report. Gut. 2007;56:772-81.

5. Malfertheiner P, Megraud F, O'Morain CA, Atherton J, Axon AT, Bazzoli F, Gensini GF, Gisbert JP, Graham DY, Rokkas T, et al. Management of Helicobacter pylori infection - the Maastricht IV/Florence Consensus Report. Gut. 2012;61:646-64.

6. Katelaris PH, Forbes GM, Talley NJ, Crotty B. A randomized comparison of quadruple and triple therapies for Helicobacter pylori eradication: the QUADRATE Study. Gastroenterology. 2002;123:1763-9.

7. Mokhtare M, Hosseini V, Tirgar Fakheri H, Maleki I, Taghvaei T, Valizadeh SM, Sardarian H, Agah S, Khalilian A. Comparison of quadruple and triple Furazolidone containing regimens on eradication of Helicobacter pylori. Med J Islam Repub Iran. 2015;29:195.

8. Pilotto A, Leandro G, Franceschi M, Rassu M, Bozzola L, Furlan F, Di Mario $F$, Valerio $G$. The effect of antibiotic resistance on the outcome of three 1-week triple therapies against Helicobacter pylori. Aliment Pharmacol Ther. 1999;13:667-73.

9. Versalovic J, Shortridge D, Kibler K, Griffy MV, Beyer J, Flamm RK, Tanaka SK, Graham DY, Go MF. Mutations in 23S rRNA are associated with clarithromycin resistance in Helicobacter pylori. Antimicrob Agents Chemother. 1996:40:477-80.

10. Fontana C, Favaro M, Minelli S, Criscuolo AA, Pietroiusti A, Galante A, Favalli C. New site of modification of 235 rRNA associated with clarithromycin resistance of Helicobacter pylori clinical isolates. Antimicrob Agents Chemother. 2002;46:3765-9.

11. Francesco VD, Zullo A, Hassan C, Giorgio F, Rosania R, lerardi E. Mechanisms of Helicobacter pylori antibiotic resistance: an updated appraisal. World J Gastrointest Pathophysiol. 2011;2:35-41.

12. van Amsterdam $K$, Bart A, van der Ende A. A Helicobacter pylori TolC efflux pump confers resistance to metronidazole. Antimicrob Agents Chemother. 2005;49:1477-82.

13. Tsugawa H, Suzuki H, Muraoka H, Ikeda F, Hirata K, Matsuzaki J, Saito Y, Hibi T. Enhanced bacterial efflux system is the first step to the development of metronidazole resistance in Helicobacter pylori. Biochem Biophys Res Commun. 2011;404:656-60.

14. Mehrabadi JF, Sirous M, Daryani NE, Eshraghi S, Akbari B, Shirazi MH. Assessing the role of the RND efflux pump in metronidazole resistance of Helicobacter pylori by RT-PCR assay. J Infect Dev Ctries. 2011;5:88-93.

15. Bina JE, Alm RA, Uria-Nickelsen M, Thomas SR, Trust TJ, Hancock RE. Helicobacter pylori uptake and efflux: basis for intrinsic susceptibility to antibiotics in vitro. Antimicrob Agents Chemother. 2000:44:248-54.

16. Hirata K, Suzuki H, Nishizawa T, Tsugawa H, Muraoka H, Saito Y, Matsuzaki J, Hibi T. Contribution of efflux pumps to clarithromycin resistance in Helicobacter pylori. J Gastroenterol Hepatol. 2010;25(Suppl 1):S75-9.

17. Binh TT, Shiota S, Suzuki R, Matsuda M, Trang TT, Kwon DH, Iwatani S, Yamaoka Y. Discovery of novel mutations for clarithromycin resistance in Helicobacter pylori by using next-generation sequencing. J Antimicrob Chemother. 2014;69:1796-803.

18. Metzker ML. Sequencing technologies - the next generation. Nat Rev Genet. 2010;11:31-46.

19. Mardis ER. Next-generation DNA sequencing methods. Annu Rev Genomics Hum Genet. 2008;9:387-402.

20. Kyrpides NC, Hugenholtz P, Eisen JA, Woyke T, Goker M, Parker CT, Amann R, Beck BJ, Chain PS, Chun J, et al. Genomic encyclopedia of bacteria and archaea: sequencing a myriad of type strains. PLoS Biol. 2014;12:e1001920.

21. Punina NV, Makridakis NM, Remnev MA, Topunov AF. Whole-genome sequencing targets drug-resistant bacterial infections. Hum Genomics. 2015;9:19.

22. Chen Y, Mukherjee S, Hoffmann M, Kotewicz ML, Young S, Abbott J, Luo Y, Davidson MK, Allard M, McDermott P, Zhao S. Whole-genome sequencing of gentamicin-resistant Campylobacter coli isolated from US retail meats reveals novel plasmid-mediated aminoglycoside resistance genes. Antimicrob Agents Chemother. 2013;57:5398-405.

23. Daum LT, Rodriguez JD, Worthy SA, Ismail NA, Omar SV, Dreyer AW, Fourie PB, Hoosen AA, Chambers JP, Fischer GW. Next-generation ion torrent sequencing of drug resistance mutations in Mycobacterium tuberculosis strains. J Clin Microbiol. 2012;50:3831-7.

24. Girault G, Blouin Y, Vergnaud G, Derzelle S. High-throughput sequencing of Bacillus anthracis in France: investigating genome diversity and population structure using whole-genome SNP discovery. BMC Genomics. 2014;15:288

25. Su P, Li Y, Li H, Zhang J, Lin L, Wang Q, Guo F, Ji Z, Mao J, Tang W, et al. Antibiotic resistance of Helicobacter pylori isolated in the Southeast Coastal Region of China. Helicobacter. 2013;18:274-9.

26. Tong YF, Lv J, Ying LY, Xu F, Qin B, Chen MT, Meng F, Tu MY, Yang NM, Li YM, Zhang JZ. Seven-day triple therapy is a better choice for Helicobacter pylori eradication in regions with low antibiotic resistance. World J Gastroenterol. 2015;21:13073-9.

27. Clinical and Laboratory Standards Institute. Performance standards for antimicrobial susceptibility testing: seventeenth informational supplement M100-S17. Wayne: CLSI; 2007.

28. Li H, Durbin R. Fast and accurate short read alignment with BurrowsWheeler transform. Bioinformatics. 2009;25:1754-60.

29. Li H, Handsaker B, Wysoker A, Fennell T, Ruan J, Homer N, Marth G, Abecasis G, Durbin R. Genome project data processing S: the sequence alignment/map format and SAMtools. Bioinformatics. 2009;25:2078-9.

30. Zagari RM, Bianchi-Porro G, Fiocca R, Gasbarrini G, Roda E, Bazzoli F. Comparison of 1 and 2 weeks of omeprazole, amoxicillin and clarithromycin treatment for Helicobacter pylori eradication: the HYPER Study. Gut. 2007;56:475-9.

31. Cao Q, Didelot X, Wu Z, Li Z, He L, Li Y, Ni M, You Y, Lin X, Li Z, et al. Progressive genomic convergence of two Helicobacter pylori strains during mixed infection of a patient with chronic gastritis. Gut. 2015;64:554-61. 
32. Kim KS, Kang JO, Eun CS, Han DS, Choi TY. Mutations in the $23 \mathrm{~S}$ rRNA gene of Helicobacter pylori associated with clarithromycin resistance. J Korean Med Sci. 2002;17:599-603.

33. Iwamoto A, Tanahashi T, Okada R, Yoshida Y, Kikuchi K, Keida Y, Murakam Y, Yang L, Yamamoto K, Nishiumi S, et al. Whole-genome sequencing of clarithromycin resistant Helicobacter pylori characterizes unidentified variants of multidrug resistant efflux pump genes. Gut Pathog. 2014;6:27.
34. Sharff A, Fanutti C, Shi J, Calladine C, Luisi B. The role of the TolC family in protein transport and multidrug efflux. From stereochemical certainty to mechanistic hypothesis. Eur J Biochem. 2001;268:5011-26.

\section{Submit your next manuscript to BioMed Central and we will help you at every step:}

- We accept pre-submission inquiries

- Our selector tool helps you to find the most relevant journal

- We provide round the clock customer support

- Convenient online submission

- Thorough peer review

- Inclusion in PubMed and all major indexing services

- Maximum visibility for your research

Submit your manuscript at

www.biomedcentral.com/submit 